Pacific Journal of Mathematics

SOME CLASSES OF EQUIVALENT GAUSSIAN PROCESSES ON 


\title{
SOME CLASSES OF EQUIVALENT GAUSSIAN PROCESSES ON AN INTERVAL
}

\author{
JACOB FELDMAN
}

1. Introduction. Let $T$ be an index set, $R, S$ real-valued nonnegative definite functions of two variables in $T$, and $m, n$ real-valued functions on $T$. Let $\Omega$ be the set of all real-valued functions on $T$, and $\mathscr{P}$ the Borel field of cylinder sets. There are then unique measures $\mu, \nu$ on $\mathscr{S}$ such that the functions $x_{t}$ on $\Omega$ defined by $x_{t}(\omega)=\omega(t)$ form Gaussian stochastic processes, with means respectively $m$ and $n$, and covariances respectively $R$ and $S$. It is shown in [2] that $\mu$ and $\nu$ are either mutually absolutely continuous or totally singular, and a necessary and sufficient condition for equivalence is given.

Suppose now that $T$ is a subset of the real line, and $R(s, t)=\imath(s-t)$, $S(s, t)=\delta(s-t)$, where, and $\&$ are continuous nonnegative-definite functions, and hence can be written as inverse Fourier transforms of finite measures $d \rho, d \sigma$. Thus, using respectively the measures $\mu$ and $\nu$ on $\Omega, x_{t}-m(t)$ and $x_{t}-n(t)$ are the restrictions to $T$ of stationary Gaussian processes on the real line. For simplicity, only the case $m=$ $n=0$ will be considered.

When $T$ is the entire real line, then it is easy to see, by looking at $d \rho$ and $d \sigma$, exactly when $\mu \sim \nu$, as is essentially known (see [3]). The precise conditions are:

a. $\rho$ and $\sigma$ must have identical non-atomic parts.

b. Their points of positive mass be the same, and if the masses are $a_{i}$ and $b_{i}$ at $x_{i}$, then $\sum\left\{\left(a_{i} / b_{i}\right)-1\right\}^{2}$ must be finite.

Now suppose $T$ is a finite interval. The problem of determining from knowledge of $\rho$ and $\sigma$ whether $\mu$ and $\nu$ are equivalent becomes much more difficult. We here discuss only a certain class of cases. Because of stationarity, one need only consider an interval symmetric about 0 . Continuity of, and \& implies that the Gaussian process is continuous with probability one at any given point, so that it makes no difference whether the interval is open or closed. There is no essential loss of generality, then, in considering only the closed interval $[-\pi, \pi]$. The following facts will then be proven:

THEOREM. Let $d \rho(x)=\left\{d x /\left(1+x^{2}\right)^{u}\right\}$, where $u$ is an integer $\geqq 1$, and let $\sigma$ be some other finite nonnegative measure on the real line. Write $\tau=\sigma-\rho$. The following conditions are necessary and sufficient that the Gaussian processes induced on $[-\pi, \pi]$ by the Fourier trans-

Received December 21, 1959, Research partly supported by Contract NONR-222 (60). 
forms of $\rho$ and $\sigma$ have equivalent measures on path space:

(a) if $k_{n}$ is a sequence of $C_{\infty}$ functions with support in $]-\pi, \pi[$ and $K_{n}$ is the Fourier transform of $k_{n}$, then $\int\left|K_{n}\right|^{2} d \sigma \rightarrow 0$ implies $\int\left|K_{n}\right|^{2} d \rho \rightarrow 0$.

(b) The Fourier transform (in the sense of Schwartz distributions) of $\left(1+x^{2}\right)^{u} d \tau(x)$ agrees on $]-2 \pi, 2 \pi[$ with a function $\psi$ such that

$$
\int_{-\pi}^{\pi} \int_{-\pi}^{\pi}|\psi(s-t)|^{2} d s d t<\infty
$$

REMARK 1. It will be seen that sufficiency still holds if (a) is weakened to:

$\left(a^{\prime}\right) \quad \int\left|K_{n}\right|^{2} d \sigma \rightarrow 0$ and $K_{n} \rightarrow K$ in $\mathscr{L}_{2}(\rho)$ implies that $K=0$ on some set of positive $\rho$-measure.

REMARK 2. As a consequence of Remark 1 , it is clear that if $\sigma$ has a component which is absolutely continuous with respect to $\rho$, then Condition (a) automatically satisfied.

Retaining the notation of the theorem:

CoRollary 1. If $d \sigma=\Phi d \rho$, where $\Phi$ is a function such that $\Phi-1$ is a finite linear combination of functions in various $L_{a}(-\infty, \infty)$ classes, $1 \leqq a \leqq 2$, then the Gaussian processes induced by $\rho$ and $\sigma$ have equivalent measures on path space.

One direction of the following corollary was proven by $D$. Slepian in [5], using techniques of G. Baxter in [1]:

COROLlaRY 2. If $A_{j}$ and $B_{1}$ are polynomials, with degrees respectively $a_{j}$ and $b_{j}, j=1,2$, and $b_{j}>a_{j}$, then the Gaussian processes whose spectral measures are $\left.\left|A_{j}(x)\right| B_{j}(x)\right|^{2} d x$ have equivalent measures on path space if and only if

(a) $b_{1}-a_{1}=b_{2}-a_{2}$

(b) the ratio of the leading coefficients of $A_{1}$ and $B_{1}$ has the same absolute value as the ratio of the leading coefficients of $A_{2}$ and $B_{2}$.

The author wishes to thank J. F. Trèves for several useful discussions about distributions.

2. Some preliminaries on functions of exponential type. First, some notation. Functions will be complex-valued functions of a real variable, unless otherwise stated. $\hat{F}$ will mean the Fourier transform of $F$ (in various degrees of generalization, depending on context), and $\check{F}$ the conjugate Fourier transform. $\sup (f)$ will mean the points where $f \neq 0$. $\mathscr{E}_{a}=$ $\{F \mid F$ extends to an entire function of exponential type $\leqq a \pi\} . \quad \mathscr{K}_{a}=$ $\mathscr{E}_{a} \cap \mathscr{L}_{2}(-\infty, \infty)$, or, by the Payley-Wiener theorem, 


$$
\begin{aligned}
& =\left\{\hat{f} \mid f \in \mathscr{L}_{2}(-\infty, \infty), \sup (f) \subset[-a \pi, a \pi]\right\} . \\
\hat{\mathscr{D}}_{a} & =\left\{f \mid f \in \mathscr{C}_{\infty}, \overline{\sup (f)} \subset\right]-a \pi, a \pi[\}, \mathscr{D}_{a}=\left\{\check{f} \mid f \in \hat{\mathscr{D}}_{a}\right\} .
\end{aligned}
$$

$u$ will be a fixed integer $\geqq 1$, and $p(x)=(i+x)^{u}$. $\rho$ is the measure $d \rho(x)=\left\{1 /|p(x)|^{2}\right\} d x$. $\mathscr{K}$ will denote the completion of $\mathscr{D}_{1}$ in the inner product $\langle F, G\rangle=\int F \bar{G} d \rho$.

Naturally, $\mathscr{K}$ really consists of equivalence classes of functions; but it will turn out that there is a continuous, in fact entire, member in each class. $H_{1}$ will denote a fixed function of $\mathscr{D}_{1}$ such that $h_{1}=\hat{H}_{1}$ is nonnegative and has integral 1. For $a>0, h_{a}(s)$ will be $(1 / a) h_{1}(s / a)$, $H_{a}(x)=H_{1}(a x)$, so that $h_{a}=\hat{H} a$, and $H_{a} \in \mathscr{D}_{a}$. Then $H_{a}$ vanishes faster than any polynomial, $\left|H_{a}(x)\right| \leqq 1$ for all $x$, and $\lim _{a \rightarrow 0} H_{a}(x)=1$ uniformly on any finite interval.

Lemma 1 . If $F \in \mathscr{E}_{1}$ and $\int|F|^{2} d \rho<\infty$, then $F \in \mathscr{K}$.

Proof. If $(1 / 2)<c<1$, then

$$
\begin{gathered}
\left(\int|F(c x)-F(x)|^{2} d \rho(x)\right)^{1 / 2} \leqq\left(\int_{-b}^{b}|F(c x)-F(x)|^{2} d \rho(x)\right)^{1 / 2} . \\
\quad+\left(\int_{|x|>b}|F(c x)|^{2} d \rho(x)\right)^{1 / 2}+\left(\int_{|x|>b}|F(x)|^{2} d \rho(x)\right)^{1 / 2} .
\end{gathered}
$$

Now,

$$
\begin{aligned}
\int_{|x|>0}|F(c x)|^{2} d \rho(x) & =\frac{1}{c} \int_{|x|>b c}|F(x)|^{2} \frac{1}{\left|p\left(\frac{x}{c}\right)^{2}\right|} d x \\
& \leqq 2 \int_{|x|<b / 2)}|F(x)|^{2} \frac{1}{|p(x)|^{2}} d x .
\end{aligned}
$$

Choosing $b$ large, and then choosing $c$ close enough to 1 to make $|F(c x)-F(x)|$ small on $[-b, b]$, we see that it suffices to show that the function $G: x \rightarrow$ $F(c x)$ is in $\mathscr{K}$. Notice that $G \in \mathscr{E}_{1}$, as $c<1$.

$H_{a} G$ is square-integrable, since $H_{a}$ vanishes faster than $\left(1 /|p|^{2}\right)$. So $H_{a} G$ is in $\mathscr{H}_{a+c}$, its Fourier transform being some $g^{\prime}$ in $\mathscr{L}_{2}(-\infty, \infty)$ with support in $[-(a+c) \pi,(a+c) \pi]$. Thus $h_{a} * g^{\prime} \in \mathscr{D}_{2 a+c}$, and $H_{a}^{2} G \in \mathscr{D}_{2 a+c}$. Choosing $a$ small causes $H_{a}^{2} G$ to be in $\mathscr{D}_{1}$, and simultaneously causes $\int\left|H_{a}^{2} G-G\right|^{2} d \rho$ to get small. This proves the lemma.

Let $\mathscr{H}=\left\{p F \mid F \in \mathscr{H}_{1}\right\}$, and $\mathscr{D}=\left\{p F \mid F \in \mathscr{D}_{1}\right\}$. Lemma 1 tells us $\mathscr{H} \subset \mathscr{K}$.

Lemma 2. $\mathscr{H}$ is precisely the closure of $\mathscr{D}$ in $\mathscr{K}$. 
Proof. First, we see that $\mathscr{H}$ is closed. If $F_{n} \in \mathscr{H}_{1}$ and

$$
\int\left|p F_{n}-G\right|^{2} d \rho \rightarrow 0 \text {, then } \int\left|F_{n}(x)-F_{m}(x)\right|^{2} d x \rightarrow 0 \text {. }
$$

Since $\mathscr{H}_{1}$ is complete, there is some $F \in \mathscr{C}_{1}$ with $\int\left|F_{n}(x)-F(x)\right|^{2} d x \rightarrow 0$. So some subsequence of the $p F_{n}$ converges almost everywhere to $p F$. Thus $p F=G$ almost everywhere.

To approximate elements $p F$ in $\mathscr{H}$ by elements in $\mathscr{D}$, just approximate $F$ in $\mathscr{L}_{2}(-\infty, \infty)$ by elements in $\mathscr{D}_{1}$, using the technique of Lemma 1.

Lemma 3. $\mathscr{K} \ominus \mathscr{H}$ is precisely the finite-dimensional space $\mathscr{L}$ of functions of the form $x \rightarrow e^{i x \pi} q(i-x)$, where $q$ is a polynomial of degree $\leqq u-1$.

Proof. Suppose $F \in \mathscr{K} \ominus \mathscr{H}$. Then $\int F \overline{p G} d \rho=0$ for all $G$ in $\mathscr{D}{ }_{1}$, i.e. $\int\{F(x) / p(x)\} \overline{G(x)} d x=0$ for all $G$ in $\mathscr{D}_{1}$. Now, $(F / p)$ is in $\mathscr{L}_{2}(-\infty, \infty)$, so it has a Fourier transform $k$ which is likewise square-integrable, and, by Plancherel's theorem, $\int k(s) \overline{g(s)} d s=0$ for all $g$ in $\hat{\mathscr{D}}_{1}$. So $k$ vanishes in $]-\pi, \pi[$.

Since $F \in \mathscr{K}, F$ can be approximated in $\mathscr{K}$ by functions $F_{n}$ in $\mathscr{D}{ }_{1}$. Each $F_{n}$ is in $\mathscr{D}_{a_{n}}$ for some $a_{n}<1$, since $\left.\overline{\sup \left(F_{n}\right)} \subset\right]-\pi, \pi$, and hence $\subset]-a_{n} \pi, a_{n} \pi$ [ for some $a_{n}<1$. Let $k_{n}$ be the Fourier transform of $F_{n} / p$. Then $k_{n} \rightarrow k$ in $\mathscr{L}_{2}(-\infty, \infty)$, and $k_{n}$ is in the domain of the $\mathscr{L}_{2}-$ differential operator $p(-i D)=i^{u}(I-D)^{u}$. So $p(-i D) k_{n}=f_{n}$, where $f_{n}$ is the Fourier transform of $F_{n}$. Since $f_{n}$ vanishes outside some $\left[-a_{n} \pi, a_{n} \pi\right], a_{n}<1$, $k_{n}$ must be of the form $\sum_{j} a_{i}^{(n)} s^{j} e^{s}$ in $]-\infty,-\pi\left[\right.$ and $\sum_{j} b_{j}^{(n)} s^{j} e^{s}$ in $] \pi, \infty[$, where $j$ ranges between 0 and $u-1$. Since $k_{n}$ is in $\mathscr{L}_{2}(-\infty, \infty)$, the $b_{j}^{(n)}$ are zero, and, letting $\phi$ be the indicator of $]-\infty,-\pi[$, we get $q k_{n}=\varphi \sum_{j} a_{j}^{(n)} s^{j} e^{s}$. This converges in $\mathscr{L}_{2}(-\infty, \infty)$, so the limit is of the form $\varphi \sum_{j} a_{j} s^{j} e^{s}$. Then $k_{n} \rightarrow 0$ in $] \pi, \infty\left[, 0\right.$ in $[-\pi, \pi]$, and $\sum_{j} a_{j} s^{j} e^{s}$ in ] $-\infty,-\pi\left[\right.$, so $k=\phi \sum_{j} a_{j} s^{j} e^{s}$. $F / p$ is then a linear combination of terms like $\int_{-\infty}^{-\pi} e^{-i x s} s^{j} e^{s} d s, 0 \leqq j \leqq u-1$, which is a linear combination of terms like $e^{i x \pi}(i+x)^{-5}, 1 \leqq j \leqq u$. Multiplying by $p$ gives the result.

Combining information from lemmas $1,2,3$ we get a description of $\mathscr{K}:$

Proposition. $\mathscr{K}$ is the orthogonal direct sum of $\mathscr{H}$ and $\mathscr{L}$.

Lemma 4. $\mathscr{D}=\mathscr{H} \cap \mathscr{D}_{1}$.

Proof. $\mathscr{D} \subset \mathscr{H}$, by definition, since $\mathscr{D}_{1} \subset \mathscr{H}_{1}$. Also $\mathscr{D} \subset \mathscr{D}_{1}$, since $\mathscr{D}_{1}$ is closed under multiplication by polynomials (because $\hat{\mathscr{D}}_{1}$ is 
closed under differentiation). So $\mathscr{D} \subset \mathscr{Y} \cap \mathscr{D}$, and it remains to show $\mathscr{D} \supset \mathscr{H} \cap \mathscr{D}_{1}$.

Suppose $G \in \mathscr{H}$. Then $G$ is a $\left\langle,>\right.$ limit of elements $G_{n}$ in $\mathscr{D}$, by Lemma 2. $G_{n}$ then has the form $p F_{n}, F_{n}$ in $\mathscr{D}_{1}$. Thus $F_{n}$ is an $\mathscr{L}_{2}(-\infty, \infty)$ Cauchy sequence, hence has a limit $F$. Then $p F=G$.

Suppose $G$ is also in $\hat{\mathscr{D}}_{1}$. Then $G$ is infinitely differentiable. Since $\hat{G}=p \hat{F}=p(-i D) \hat{F}$, we conclude that $\hat{F}$ is infinitely differentiable. Now

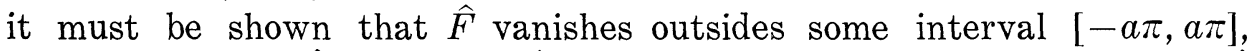
$0<a<1$. But $\hat{G}=p(-i D) \hat{F}$ vanishes outside such on interval, so $\hat{F}$ is analytic outside $[-a \pi, a \pi]$. Also, $\hat{F}$ vanishes outside $[-\pi, \pi]$, since each $\hat{F}_{n}$ has support in $]-\pi, \pi[$. Therefore, $\hat{F}$ vanishes outside $[-a \pi$, $a \pi]$. So $\hat{F}$ is in $\mathscr{D}_{1}$, and $F$ is in $\mathscr{D}_{1}$.

Lemma 5. $\mathscr{D}_{1} / \mathscr{D}$ is finite dimensional.

Proof. $\mathscr{D}_{1}\left|\mathscr{D}=\mathscr{D}_{1}\right| \mathscr{D}_{1} \cap \mathscr{H} \approx\left(\mathscr{D}_{1}+\mathscr{K}\right)|\mathscr{H} \subset \mathscr{Y}| \mathscr{H} \approx \mathscr{L}$.

3. Proof of theorem. In [2] it is shown that a necessary and sufficient condition for equivalence of $\mu$ and $\nu$ is that there be an equivalence operator from the closed linear span of $\left\{x_{t} \mid t \in T\right\}$ in $\mathscr{L}_{2}(\mu)$ to their closed linear span in $\mathscr{L}_{2}(\nu)$, sending the $\mu$-equivalence class of $x_{t}$ to the $\nu$-equivalence class of $x_{t}$ for each $t \in T$. (An equivalence operator, as defined in [2], is a linear homeomorphism $H$ between two Hilbert spaces such that $I-H^{*} H$ is Hilbert Schmidt). Actually, we shall want the condition in complex $\mathscr{L}_{2}$, while the proof in [2] is for real $\mathscr{L}_{2}$; however, the transition from the one to the other is immediate.

Under this condition, $H$ would map $\int_{-\pi}^{\pi} f\left(x_{t}\right) d t$ as an $\mathscr{L}_{2}(\mu)-$ valued integral to $\int_{-\pi}^{\pi} f(t) x_{t} d t$ as an $\mathscr{L}_{2}(\nu)$-valued integral, for each $f \in \hat{\mathscr{D}}_{1}$; and conversely, if $H$ had this effect on all such $\int_{-\pi}^{\pi} f(t) x_{t} d t$, then by choosing a sequence of $f$ approximating a delta function, one could verify that $H$ sent the equivalence class of $x_{t}$ in $\mathscr{L}_{2}(\mu)$ to the equivalence class of $x_{t}$ in $\mathscr{L}_{2}(\nu)$. Therefore, putting inner products (, ) and (, ) on $\hat{\mathscr{D}}_{1}$ by the rules

$$
\begin{aligned}
& (f, g)=\int_{-\pi}^{\pi} \int_{-\pi}^{\pi} i(s-t) f(s) \overline{g(t)} d s d t, \\
& (f, g)^{\cdot}=\int_{-\pi}^{\pi} \int_{-\pi}^{\pi},(s-t) f(s) g \overline{(t) d} s d t,
\end{aligned}
$$

and noting that $(f, g)=\int\left(\int_{-\pi}^{\pi} f(s) x_{s} d s\right)\left(\int_{-\pi}^{\pi} g(t) x_{t} d t\right) d \mu$ and

$$
(f, g)^{\cdot}=\int\left(\int_{-\pi}^{\pi} f(s) x_{s} d s\right)\left(\int_{-\pi}^{\pi} g(t) x_{t} d t\right) d v,
$$

it follows that a necessary and sufficient condition for the equivalence of $\mu$ and $\nu$ is the existence of an equivalence operator from the (, ) com- 
pletion of $\hat{\mathscr{D}}_{1}$ to its $(,)^{\cdot}$ completion, and sending the (, )-equivalence class of $f$ to its $(,)^{\circ}$-equivalence class.

Now let $\langle F, G\rangle^{\bullet}=\int F \bar{G} d \sigma$, where $F$ and $G$ are in $\mathscr{D}_{1}$ (and hence continuous and bounded, so that the integral exists). Let $\mathscr{\mathscr { K }}$ be the closure of $\mathscr{D}_{1}$ in $\mathscr{L}_{2}(\sigma)$. Let $J$ be the map assigning to $F$ in $\mathscr{D}_{1}$ its equivalence class in $\dot{\mathscr{K}}$. Since $\langle F, G\rangle=(\hat{F}, \widehat{G})$, and $\langle F, G\rangle=(\hat{F}, \widehat{G})^{\circ}$, the necessary and sufficient condition for the equivalence of $\mu$ and $\nu$ in the theorem is that $J$ be the restriction to $\mathscr{D}_{1}$ of an equivalence map from $\mathscr{K}$ to $\mathscr{\mathscr { K }}$.

To prove sufficiency of the conditions in the theorem, suppose first that $|p(x)|^{2} d \tau(x)$ has a generalized Fourier transform (see [4]) which agrees on $]-2 \pi, 2 \pi\left[\right.$ with a function $\psi$ such that $\int_{-\pi}^{\pi} \int_{-\pi}^{\pi}|\psi(s-t)|^{2} d s d t=$ $a^{2}<\infty$. We extend $\psi$ by making it 0 outside $]-2 \pi, 2 \pi[$.

\section{Lemma 6. If $F \in \mathscr{D}$, then $\langle F, F\rangle \cdot \leqq(1+a)\langle F, F\rangle$.}

Proof: Write $F=p G, G \in \mathscr{D}_{1}$. Then $\int|F|^{2} d \sigma=\int|F|^{2} d \rho+\int|G|^{2}|p|^{2} d \tau$. Now, $\hat{G}$ is in $\hat{\mathscr{D}}_{1}$, so $\hat{G} * \hat{G}$ is infinitely differentiable with support in ]$-2 \pi, 2 \pi[$. Then, by Schwartz's definition of generalized Fourier transform, we get $\int|G|^{2}|p|^{2} d \tau=\int_{-2 \pi}^{2 \pi} \hat{G} * \hat{G}(s) \psi(s) d s=\int_{-2 \pi}^{2 \pi} \int_{a(s)}^{b(s)} \hat{G}(s-t) \overline{\hat{G}}(1-t)$ $\psi(s) d t d s$, where $a(s)=\max (-\pi, s-\pi)$ and $b(s)=\min (\pi, s+\pi)$. Letting $s-t=s^{\prime}$, and $t=-t^{\prime}$ gives $\int_{-\pi}^{\pi} \int_{-\pi}^{\pi} \hat{G}\left(s^{\prime}\right) \hat{G}\left(t^{\prime}\right) \psi\left(s^{\prime}-t^{\prime}\right) d s^{\prime} d t^{\prime}$, whose absolute value, by the Schwartz inequality, is

$$
\begin{aligned}
& \leqq\left[\int_{-\pi}^{\pi} \int_{-\pi}^{\pi}|\hat{G}(s) \overline{\hat{G}(t)}|^{2} d s d t \int_{-\pi}^{\pi} \int_{-\pi}^{\pi}|\psi(s-t)|^{2} d s d t\right]^{1 / 2} \\
& =\left(\int_{-\pi}^{\pi}|\hat{G}(s)|^{2} d s\right) a=\left(\int|F|^{2} d \rho\right) a .
\end{aligned}
$$

Pick a complete orthonormal set (c.o.n.s.) $f_{1}, f_{2}, \cdots$ for $\mathscr{L}_{2}(-\pi, \pi)$ out of the dense subset $\hat{\mathscr{D}}_{1}$. Let $F_{n}=\check{f}_{n}$, and $G_{n}=p F_{n}$. Then the $G_{n}$ form a c.o.n.s. for $\mathscr{H}$ (in $\langle$,$\rangle ) consisting of elements of \mathscr{D}$, because the $F_{n}$ are a c.o.n.s. for $\mathscr{H}_{1}$ consisting of elements of $\mathscr{D}_{1}$.

LEMMA 7. $\sum_{n, m=1}^{\infty}\left|\left\langle G_{n}, G_{m}\right\rangle-\left\langle G_{n}, G_{m}\right\rangle \cdot\right|^{2}=a^{2}$.

Proof. $\int G_{n}(x) \overline{G_{m}(x)} d \tau(x)=\int_{-2 \pi}^{2 \pi} \hat{F}_{n} * \hat{F}_{m}(s) \psi(s) d s$. By using a change of variable as in the previous lemma, this equals $\int_{-\pi}^{\pi} \int_{-\pi}^{\pi} f_{n}(s) \overline{f_{m}(t)} \psi(s-t) d s d t$. But the functions $(s, t) \rightarrow \overline{\left(f_{n} s\right)} f_{m}(t)$ form a c.o.n.s. in $\mathscr{L}_{2}([-\pi, \pi] \times[-\pi, \pi])$, so that $\sum_{n, m=1}^{\infty}\left|\int_{-\pi}^{\pi} \int_{-\pi}^{\pi} f_{n}(s) \overline{f_{m}(t)} \psi(s-t) d s d t\right|^{2}$ is exactly $\int_{-\pi}^{\pi} \int_{-\pi}^{\pi}|\psi(s-t)|^{2} d s d t$.

Now consider the map $J$ from $\mathscr{D}_{1}$ to $\dot{\mathscr{K}}$. Lemma 6 implies that its restriction to $\mathscr{D}$ is bounded, and, since $\mathscr{D}{ }_{1} / \mathscr{D}$ is finite-dimensional (Lemma 5$), J$ is bounded as an operator from $\mathscr{D}_{1}$ to $\dot{\mathscr{K}}$ (a finite-dimensional 
extension of a bounded operator is bounded, as is readily seen). So $J$ extends uniquely to a bounded operator $A$ from $\mathscr{K}$ to $\mathscr{\mathscr { K }}$.

Lemma 8. $I-A^{*} A$ is a Hilbert-Schmidt operator.

Proof. Complete the o.n.s. $G_{1}, G_{2}, \cdots$ by adding to it a c.o.n.s. $G_{0}, G_{-1}, \cdots, G_{1-u}$ in $\mathscr{L}$. Then, letting $k=u-1$,

$$
\begin{aligned}
& \sum_{n, m=-k}^{\infty}\left|\left\langle\left(I-A^{*} A\right) G_{n}, G_{m}\right\rangle\right|^{2} \\
&= \sum_{n, m=1}^{\infty}\left|\left\langle\left(I-A^{*} A\right) G_{n}, G_{m}\right\rangle\right|^{2} \\
&+\sum_{n=-k}^{0} \sum_{m=-k}^{\infty}\left|\left\langle\left(I-A^{*} A\right) G_{n}, G_{m}\right\rangle\right|^{2} \\
&+\sum_{n=-k}^{\infty} \sum_{m=-k}^{0}\left|\left\langle G_{n},\left(I-A^{*} A\right) G_{m}\right\rangle\right|^{2} \\
&= \sum_{n, m=1}^{\infty}\left|\left\langle G_{n}, G_{m}\right\rangle-\left\langle A G_{n}, A G_{m}\right\rangle \cdot\right|^{2} \\
&+\left.2 \sum_{n=-k}^{0}\left|\left\langle I-A^{*} A\right) G_{n},\left(I-A^{*} A\right) G_{n}\right\rangle\right|^{2},
\end{aligned}
$$

using Parseval's equality. But $\left\langle A G_{n}, A G_{m}\right\rangle \cdot=\left\langle G_{n}, G_{m}\right\rangle \cdot$ for $\left.n, m\right\rangle 0$, since such $G_{n}$ are in $\mathscr{D}_{1}$, so that the sum is exactly

$$
a^{2}+2 \sum_{n=-k}^{0}\left\langle\left(I-A^{*} A\right) G_{n},\left(I-A^{*} A\right) G_{n}\right\rangle .
$$

In order to complete the proof, it must be shown that $A$ is a homeomorphism from $\mathscr{K}$ onto $\mathscr{\mathscr { K }}$. Since $I-A^{*} A$ is completely continuous, it will suffice to show

(1) that the range of $A$ is dense in $\dot{\mathscr{K}}$.

(2) that $A$ sends no nonzero element to zero.

(1) is clear, since the range of $A$ contains the range of $J$, which is dense by the very definition of $\dot{\mathscr{K}}$.

We now make use of (a), or rather of the weaker $\left(a^{\prime}\right)$, to prove (2). Suppose, in fact, that $A(K)$ is zero in $\dot{\mathscr{K}}$ for some $K$ in $\mathscr{K}$. Let $K_{n}$ be a sequence of members of $\mathscr{D}_{1}$ converging to $K$ in $\mathscr{K}$. Then $K_{n}$ converges to zero in $\dot{K}$, since $A\left(K_{n}\right)=J\left(K_{n}\right)$. Then, by $\left(\mathrm{a}^{\prime}\right)$, $K=0$ on a set of positive $\rho$ measure. But the Proposition of the previous section tells us that $K$ is analytic. Thus $K=0$.

To show the necessity of condition (a), suppose $J$ has an extension to an equivalence operator from $\mathscr{K}$ to $\dot{\mathscr{K}}$, which we call $A$. Then (a) is immediate from the fact that $A$ is continuously invertible.

Since $I-A^{*} A$ is an equivalence operator, $\sum_{n, m=1}^{\infty} \mid\left\langle G_{n}, G_{m}\right\rangle-$ $\left.\left\langle G_{n} G_{m}\right\rangle \cdot\right|^{2}<\infty$, where $G_{1}, G_{2}, \cdots$ is the c.o.n.s. in $\mathscr{D}$ for $\mathscr{C}$ previously constructed. Define an operator $Z$ on $\mathscr{L}_{2}([-\pi, \pi] \times[-\pi, \pi])$ as follows : let $f_{n, m}(s, t)=f_{n}(s) f_{m}(t)$, where $G_{n}=p \check{f}_{n}$. For $Q=\sum_{n, m} a_{n, m} f_{n, m}$, Let $Z(Q)=\sum_{n, m} a_{n, m}\left(\left\langle G_{n}, G_{m}\right\rangle-\left\langle G_{n}, G_{m}\right\rangle \cdot\right)$. Then

$$
|Z(Q)|^{2} \leqq \sum_{n, m}\left|a_{n, m}\right|^{2} \sum_{n, m}\left|\left\langle G_{n}, G_{m}\right\rangle-\left\langle G_{n}, G_{m}\right\rangle \cdot\right|^{2} .
$$


So $Z(Q)$ has the form $\int_{-\pi}^{\pi} \int_{-\pi}^{\pi} Q(s, t) \Psi(s, t) d s d t$ for some $\Psi$ such that $\int_{-\pi}^{\pi} \int_{-\pi}^{\pi}|\Psi(s, t)|^{2} d s d t<\infty$. In particular, consider $f, g \in \mathscr{D}_{1}$, and let $f=$ $\sum_{n} a_{n} f_{n}, g=\sum b_{m} f_{m}$. Let $Q(s, t)=f(s) \overline{g(t)}$. Then $Z(Q)=\sum_{n, m} a_{n} b_{m}\left\langle\left\langle G_{n}, G_{m}\right\rangle\right.$ $\left.-\left\langle G_{n}, G_{m}\right\rangle\right)=\sum_{n, m} a_{n} \bar{b}_{m} \int\left(p F_{n}\right)\left(\overline{p F_{m}}\right) d t=\int \check{f} \check{g} \bar{g}|p|^{2} d t$.

Let $0<r<2 \pi$, and let $f, g$ have the closure of their supports in in $]-\pi+r, \pi\left[\right.$. Let $f^{\prime}(s)=f(s+r), g^{\prime}(s)=g(s+r)$. Then $f^{\prime}, g^{\prime}$ are in $\mathscr{D}_{1}$, and their inverse Fourier transforms satisfy $\check{f}^{\prime}(x)=e^{i r x} \check{f}(x)=e^{i r x} \check{g}(x)$. Then

$$
\begin{aligned}
\int_{-\pi}^{\pi} \int_{-\pi}^{\pi} f^{\prime}(s) \overline{g^{\prime}(t)} \Psi(s, t) d s d t=\int \check{f}^{\prime} \bar{g}^{\prime}|p|^{2} d t \\
=\int f \bar{g}|p|^{2} d t=\int_{-\pi}^{\pi} \int_{-\pi}^{\pi} f(s) \overline{g(t)} \Psi(s, t) d s d t .
\end{aligned}
$$

But

$$
\int_{-\pi}^{\pi} \int_{-\pi}^{\pi} f(s+r) \overline{g(t+r)} \Psi(s, t) d s d t=\int_{-\pi}^{\pi} \int_{-\pi}^{\pi} f(s) \overline{g(t)} \psi(s-r, t-r) d s d t .
$$

in view of the restrictions on the support of $f$ and $g$. Since this holds for all such $f, g$, the equality $\Psi(s-r, t-r)=\Psi(s, t)$ holds for almost all $(s, t)$ for which $s, t, s-r, t-r$ are in $]-\pi, \pi[$ ( $r$ being fixed). Thus, $\{(r, s, t) \mid s, t, s-r$, are in $]-\pi, \pi[$ and $\Psi(s-r, t-r) \neq \Psi(s, t)\}$ has measure zero.

Applying Fubini's theorem, we get: for almost all pairs $s, t$ in $]-\pi, \pi[$ the set $\{r \mid s-r, t-r$ lie in $]-\pi, \pi[$ and $\Psi(s-r, t-r) \neq \Psi(s, r)\}$ has measure 0 . Denote by $\Delta$ the exceptional set of pairs $(s, t)$.

Now let $\Gamma_{s}$ be the line of slope 1 which passes through $(s,-s)$, where $-\pi<s<\pi$. Let $\Gamma$ be the set of $s$ for which $\Gamma_{s} \cap \Delta$ is not a set of measure 0 . Then $\Gamma$ has measure 0, again by Fubini's theorem, and by rotation-invariance of Lebesgue measure. If $s$ is in $]-\pi, \pi[$ but not in $I$, then almost all points on that portion of $L_{s}$ which lies in ]$-\pi, \pi[\times]-\pi, \pi[$ assign to $\Psi$ a common value; thus, if the function $\Psi^{\prime}$ is defined on $]-\pi, \pi\left[\right.$ by $\Psi^{\prime}(s, t)=\int_{a(s, t)}^{b(s, t)} \Psi(s-r, t-r) d r$, where $a(s, t)=$ $\max (s-\pi, t-\pi)$ and $b(s, t)=\min (s+\pi, t+\pi)$, then, for $(s, t)$ on $\Gamma_{r}$, $\Psi^{\prime}(s, t)$ has this common value. Thus, for almost all $r, \Psi^{\prime}(s, t)=\Psi(s, t)$ for almost all (in linear measure) points $(s, t)$ with $-\pi<s, t<\pi$ and $s$, $t$ on $\Gamma_{r}$. Then $\Psi^{\prime}(s, t)$ is equal almost everywhere to $\Psi(s, t)$. Now set $\psi(r)=\Psi(-r / 2, r / 2),-2 \pi<r<2 \pi$.

Then

$$
\begin{aligned}
\Psi^{\prime}(s, t)= & \Psi^{\prime}(s-(s+t) / 2, t-(s+t) / 2) \\
& =\Psi^{\prime}(-(t-s) / 2,(t-s) / 2)=\psi(t-s),
\end{aligned}
$$

for $s, t$ in $]-\pi, \pi[$. This completes the proof.

Corollary 1 is just a consequence of the fact (proven in [4]) that if 
$\Phi$ is as in the statement, then $(\bar{\Phi}-1) d x$ has a generalized Fourier transform which is a function $\varphi$ square-summable in any finite interval, so that

$$
\int_{-\pi}^{\pi} \int_{-\pi}^{\pi}|\varphi(s-t)|^{2} d s d t \leqq\left.\left|\int_{-\pi}^{\pi} \int_{-2 \pi}^{2 \pi}\right| \varphi(r)\right|^{2} d r \leqq 2 \pi \int_{-2 \pi}^{2 \pi}|\varphi(r)|^{2} d r .
$$

To prove corollary 2: let $c_{j}$ be the absolute value of the ratio of the leading terms of $A_{j}$ and $B_{j}$, and let $u_{j}=b_{j}-a_{j}=\operatorname{deg}\left(B_{j}\right)-\operatorname{deg}\left(A_{j}\right)$. It is clear in general that equivalence of the Gaussian processes induced by given covariances is unaffected if both covariances are multiplied by the same constant. Thus, we find that the process whose spectral measure is

$$
\left|\frac{A_{j}(x)}{B_{j}(x)}\right|^{2} d x
$$

has measure on path space equivalent to that whose spectral measure is

$$
\frac{c_{j}}{\left(1+x^{2}\right)^{u_{j}}} d x
$$

because the quotient of

$$
\left|\frac{A_{j}(x)}{B_{j}(x)}\right|^{2} \quad \text { by } \quad \frac{c_{j}}{\left(1+x^{2}\right)^{u_{i}}}
$$

is of the form: 1 plus a function in $\mathscr{L}_{2}(-\infty, \infty)$. So the problem is reduced to whether or not the processes with spectral measures

$$
\frac{1}{\left(1+x^{2}\right)^{u_{1}}} d x \text { and } \frac{c_{2} c_{1}^{-1}}{\left(1+x^{2}\right)^{u_{2}}} d x
$$

are equivalent. The criterion is that

$$
\left(1-\frac{c_{2} c_{1}^{-1}}{\left(1+x^{2}\right)^{u_{2}-u_{1}}}\right) d x
$$

have a generalized Fourier transform which agrees with a function on ]$-2 \pi, 2 \pi$ [ having certain properties. But this generalized Fourier transform is explicitly calculated (see [4]), and is of the required form when and only when $c_{2}=c_{1}$ and $u_{2}=u_{1}$.

\section{BIBLIOGRAPHY}

1. G. Baxter, A strong limit theorem for Gaussian processes, Proc. Amer. Math. Soc., (1956).

2. J. Feldman, Equivalence and perpendicularity of Gaussian Processes, Pacific J. Math., 8, No. 4, (1958). 
3. U. Grenander, Stochastic processes and statistical inference, Arkiv furti Matemak, Band 1 , nr. 17.

4. L. Schwartz, Théorie des distributions: Hermann and $C^{i e}$, Paris, 1951.

5. D. Slepian, Some comments on the detections of Gaussian signals in Gaussian noise: I. R. E. Transactions on Information Theory, Vol. I-4, June, 1958.

\section{UNIVERSITY OF CALIFORNIA, BERKELEY}




\section{PACIFIC JOURNAL OF MATHEMATICS}

\section{EDITORS}

David GILbarg

Stanford University

Stanford, California

\section{F. H. BRowneLL}

University of Washington

Seattle 5, Washington

\section{A. L. Whiteman}

University of Southern California Los Angeles 7, California

\section{J. PAIGe}

University of California

Los Angeles 24, California

\section{ASSOCIATE EDITORS}
E. F. BECKENBACH
T. M. CHERRY
D. DERRY

\author{
E. HEWITT \\ A. HORN \\ L. NACHBIN
}

\author{
M. OHTSUKA \\ H. L. ROYDEN \\ M. M. SCHIFFER
}

E. SPANIER

E. G. STRAUS

F. WOLF

\section{SUPPORTING INSTITUTIONS}

\author{
UNIVERSITY OF BRITISH COLUMBIA \\ CALIFORNIA INSTITUTE OF TECHNOLOGY \\ UNIVERSITY OF CALIFORNIA \\ MONTANA STATE UNIVERSITY \\ UNIVERSITY OF NEVADA \\ NEW MEXICO STATE UNIVERSITY \\ OREGON STATE COLLEGE \\ UNIVERSITY OF OREGON \\ OSAKA UNIVERSITY \\ UNIVERSITY OF SOUTHERN CALIFORNIA
}

\author{
STANFORD UNIVERSITY \\ UNIVERSITY OF TOKYO \\ UNIVERSITY OF UTAH \\ WASHINGTON STATE COLLEGE \\ UNIVERSITY OF WASHINGTON \\ AMERICAN MATHEMATICAL SOCIETY \\ CALIFORNIA RESEARCH CORPORATION \\ HUGHES AIRCRAFT COMPANY \\ SPACE TECHNOLOGY LABORATORIES \\ NAVAL ORDNANCE TEST STATION
}

\footnotetext{
Mathematical papers intended for publication in the Pacific Journal of Mathematics should be typewritten (double spaced), and the author should keep a complete copy. Manuscripts may be sent to any one of the four editors. All other communications to the editors should be addressed to the managing editor, L. J. Paige at the University of California, Los Angeles 24, California.

50 reprints per author of each article are furnished free of charge; additional copies may be obtained at cost in multiples of 50 .
}

The Pacific Journal of Mathematics is published quarterly, in March, June, September, and December. The price per volume (4 numbers) is $\$ 12.00$; single issues, $\$ 3.50$. Back numbers are available. Special price to individual faculty members of supporting institutions and to individual members of the American Mathematical Society: $\$ 4.00$ per volume; single issues, $\$ 1.25$.

Subscriptions, orders for back numbers, and changes of address should be sent to Pacific Journal of Mathematics, 2120 Oxford Street, Berkeley 4, California.

Printed at Kokusai Bunken Insatsusha (International Academic Printing Co., Ltd.), No. 6, 2-chome, Fujimi-cho, Chiyoda-ku, Tokyo, Japan.

PUBLISHED BY PACIFIC JOURNAL OF MATHEMATICS, A NON-PROFIT CORPORATION

The Supporting Institutions listed above contribute to the cost of publication of this Journal, but they are not owners or publishers and have no responsibility for its content or policies. 


\section{Pacific Journal of Mathematics}

\section{Vol. 10, No. $4 \quad$ December, 1960}

M. Altman, An optimum cubically convergent iterative method of inverting a linear bounded operator in Hilbert space . . . . . . . . . . . . . . . . . . . . . . . . . . 1107

Nesmith Cornett Ankeny, Criterion for rth power residuacity ................. 1115

Julius Rubin Blum and David Lee Hanson, On invariant probability measures I . . . . . 1125

Frank Featherstone Bonsall, Positive operators compact in an auxiliary topology ..... 1131

Billy Joe Boyer, Summability of derived conjugate series . . . . . . . . . . . . . . . . 1139

Delmar L. Boyer, A note on a problem of Fuchs . . . . . . . . . . . . . . . . . 1147

Hans-Joachim Bremermann, The envelopes of holomorphy of tube domains in infinite

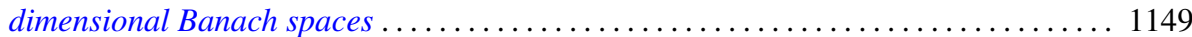

Andrew Michael Bruckner, Minimal superadditive extensions of superadditive

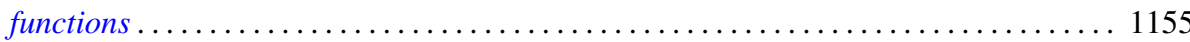

Billy Finney Bryant, On expansive homeomorphisms .................... 1163

Jean W. Butler, On complete and independent sets of operations in finite algebras . . . . . 1169

Lucien Le Cam, An approximation theorem for the Poisson binomial distribution ...... 1181

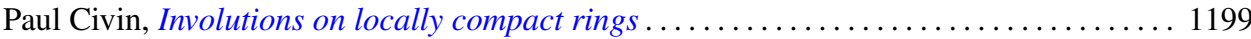

Earl A. Coddington, Normal extensions of formally normal operators . . . . . . . . . 1203

Jacob Feldman, Some classes of equivalent Gaussian processes on an interval ........ 1211

Shaul Foguel, Weak and strong convergence for Markov processes . . . . . . . . . . . 1221

Martin Fox, Some zero sum two-person games with moves in the unit interval ........ 1235

Robert Pertsch Gilbert, Singularities of three-dimensional harmonic functions . . . . . . . 1243

Branko Grünbaum, Partitions of mass-distributions and of convex bodies by

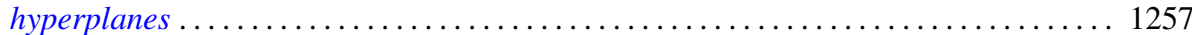

Sidney Morris Harmon, Regular covering surfaces of Riemann surfaces ........... 1263

Edwin Hewitt and Herbert S. Zuckerman, The multiplicative semigroup of integers

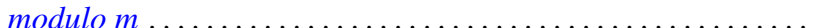

Paul Daniel Hill, Relation of a direct limit group to associated vector groups . ......... 1309

Calvin Virgil Holmes, Commutator groups of monomial groups . .

James Fredrik Jakobsen and W. R. Utz, The non-existence of expansive homeomorphisms

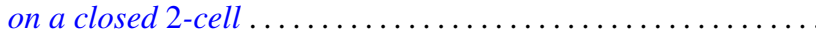

John William Jewett, Multiplication on classes of pseudo-analytic functions . . . . . . . 1323

Helmut Klingen, Analytic automorphisms of bounded symmetric complex domains . . . . 1327

Robert Jacob Koch, Ordered semigroups in partially ordered semigroups . . . . . . . . 1333

Marvin David Marcus and N. A. Khan, On a commutator result of Taussky and

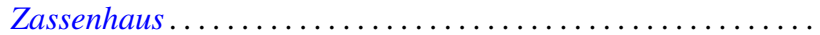

John Glen Marica and Steve Jerome Bryant, Unary algebras......

Edward Peter Merkes and W. T. Scott, On univalence of a continued fraction . . . . . . . 1361

Shu-Teh Chen Moy, Asymptotic properties of derivatives of stationary measures . . . . . 1371

John William Neuberger, Concerning boundary value problems . . . . . . . . . . . 1385

Edward C. Posner, Integral closure of differential rings . . . . . . . . . . . . . . . . . 1393

Marian Reichaw-Reichbach, Some theorems on mappings onto . . . . . . . . . . . . . 1397

Marvin Rosenblum and Harold Widom, Two extremal problems . . . . . . . . . . . . . . . . 1409

Morton Lincoln Slater and Herbert S. Wilf, A class of linear differential-difference

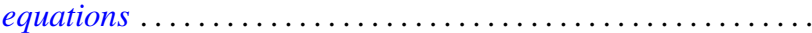

Charles Robson Storey, Jr., The structure of threads . . . . . . . . . . . . . . . . . . 1429

J. François Treves, An estimate for differential polynomials in $\partial / \partial z_{1},, \cdots, \partial / \partial z_{-} n \ldots \ldots 1447$

J. D. Weston, On the representation of operators by convolutions integrals . . . . . . . . 1453

James Victor Whittaker, Normal subgroups of some homeomorphism groups ......... 1469 